\title{
Spin-Forbidden Channels in Reactions of Unsaturated Hydrocarbons with $\mathrm{O}\left({ }^{3} \mathrm{P}\right)$
}

\author{
Pavel Pokhilko ${ }^{a}$, Robin Shannon ${ }^{b}$, David Glowacki ${ }^{b}$, \\ Hai Wang $^{c}$, and Anna I. Krylov ${ }^{a}$ \\ ${ }^{a}$ Department of Chemistry, University of Southern California, Los Angeles, California 90089-0482, U.S.A. \\ ${ }^{b}$ School of Chemistry, University of Bristol, Cantock's Close, Bristol BS8 1TS, U.K. \\ ${ }^{c}$ Department of Mechanical Engineering, Stanford University, Stanford, California 94305-3032, U.S.A.
}

October 19, 2018

\begin{abstract}
Electronic structure of four prototypical Cvetanović diradicals, species derived by addition of $\mathrm{O}\left({ }^{3} \mathrm{P}\right)$ to unsaturated compounds, is investigated by high-level electronic structure calculations and kinetics modeling. The main focus of this study is on the electronic factors controlling the rate of inter-system crossing (ISC), minimal energy crossing points (MECPs) and spin-orbit couplings (SOCs). The calculations illuminate significant differences in the electronic structure of ethylene- and acetylene-derived compounds and a relatively minor effect due to methylation. The computed MECPs heights and SOCs reveal different mechanisms of ISC in ethylene- and acetylene-derived species, thus explaining variations in the observed branching ratios between singlet and triplet products and a puzzling effect of the methyl substitution. In the ethylene- and propylene-derived species, the MECP is very low and the rate is controlled by the SOC variations, whereas in the acetylene- and propyne-derived species the MECP is high and the changes in the ISC rate due to methyl substitutions are driven by the variations in MECP heights.
\end{abstract}

\section{Introduction}

Among various reaction products, triplet oxygen atom reacting with unsaturated hydrocarbons $(\mathrm{RH})$ can produce Cvetanović diradicals, ${ }^{1,2}$ species in which $\mathrm{O}\left({ }^{3} \mathrm{P}\right)$ is added to a double or a triple bond. Because oxygen is triplet, the diradical is formed in the triplet state. It can then undergo series of reactions on the triplet potential energy surface (PES) as well as intersystem crossing (ISC) to the singlet manifold. The reactions of Cvetanović diradicals are important in hydrocarbon combustion, and, to an extent, in atmospheric and interstellar chemistry. ${ }^{1-5}$ For example, the reaction of ethylene with $\mathrm{O}\left({ }^{3} \mathrm{P}\right)$ can lead to effective secondary chain-branching and thus enhanced fuel oxidation rate. Although numerous studies have investigated the role of Cvetanovic diradicals and ISC in the reactions of $\mathrm{O}\left({ }^{3} \mathrm{P}\right)$ with unsaturated hydrocarbons, ${ }^{3,4,6-15}$ the key details of ISC are still unresolved. One particularly interesting question is what aspects of electronic structure of the Cvetanović diradicals derived from different unsaturated 
hydrocarbons control the rate of ISC. Fig. 1 shows the yields of singlet reaction products in the reaction of $\mathrm{O}\left({ }^{3} \mathrm{P}\right)$ with ethylene, ${ }^{16}$ acetylene, ${ }^{17}$ propene,,${ }^{18}$ and propyne. ${ }^{19}$ In these four unsaturated hydrocarbon species, the branching ratios between the singlet and triplet pathways vary from $10 \%$ to $84 \%$. The efficiency of ISC in alkenes and alkynes is quite different: while in ethylene the branching ratio for the singlet products is above $50 \%$, in acetylene it is less than $10 \%$. Most intriguing are variations due to substituting one of the hydrogens by methyl group: in alkenes, this substitution results in a moderate drop of the singlet products yield, but in alkynes the branching ratio increases eightfold. The goal of the present study is to characterize the electronic structure of the prototypical Cvetanovic diradicals derived by $\mathrm{O}\left({ }^{3} \mathrm{P}\right)$ addition to ethene, propene, acetylene, and propyne, with an emphasis on factors relevant to ISC. While the quantitative determination of the yield of the reaction products formed via ISC to the singlet state requires full multi-well modeling of the reaction ${ }^{20,21}$ at the conditions that match the available experiments, the rate of the actual ISC step is determined by two quantities ${ }^{15,22-25}$ - spin-orbit coupling constant (SOCC) and the location of minimal-energy crossing point (MECP).
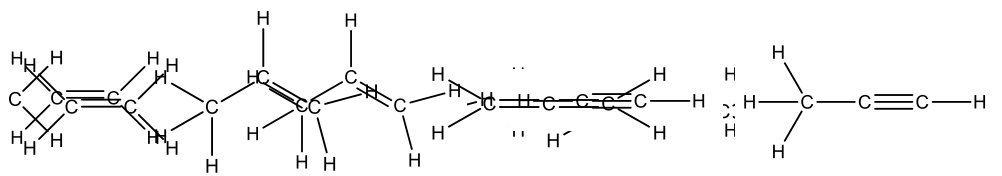

Ethene (>50\%)

Propene (40\%) Acetylene (<10\%)

Propyne (84\%)

Figure 1: Total yields of the reaction products via ISC in ethene, propene, acetylene, and propyne.

Electronic structure of $\mathrm{C}_{2} \mathrm{H}_{4} \mathrm{O}$ has been extensively studied before. ${ }^{4,9,11,14,15,26}$ The ISC has been studied by Bowman and co-workers, by surface-hopping, ${ }^{9,10}$ and by Klippenstein and co-workers, by non-adiabatic transition-state theory (NA-TST) and dynamical simulations. ${ }^{4,15}$ While most these ISC calculations were carried out for only a single triplet surface, Ref. 15 considered the contribution to the rate from 2 triplet states. The simulations revealed that ISC in Cvetanović diradicals can indeed be very effective and showed that different treatments of ISC yield different kinetic predictions at high temperature. In this work we explore the qualitative differences between the electronic structure of several Cvetanović diradicals and evaluate important trends and features relevant to ISC. We focus on general trends rather than on quantitative rates modeling. The paper outline is as follows. In the next section, we describe molecular orbital (MO) framework and the nature of relevant electronic states. Section 3 outlines theoretical methods employed and computational details. Results of electronic structure calculations as well as their implication for kinetics are presented in Section 4.

\section{Molecular orbital framework}

Electronic structure of the Cvetanović diradicals is complex, featuring a manifold of closely lying electronic states derived by distributing 4 electrons over 3 orbitals. To simplify the description of electronic states, in the case of ethylene adduct we considered a symmetric geometry of the lowest triplet state (with the CCO symmetry plane), as was done in previous studies $^{7,26}$ (the structures and relevant energetics is shown in Fig. S1 in the SI). Figure 2 shows 
frontier MOs of $\mathrm{C}_{2} \mathrm{H}_{4} \mathrm{O}$ at the optimized geometry of the ${ }^{3} \mathrm{~A}^{\prime \prime}$ state and the leading electronic configurations of the relevant states. Although the exact shape of orbitals depends on the method, their characters can be reliably described as out-of-plane lone pair on oxygen, $\operatorname{lp}(\mathrm{O})$, and bonding-antibonding pair formed by in-plane $p$-orbital of oxygen and $p$-orbital of carbon. Different electronic distributions of 4 electrons on these 3 orbitals give rise to several closely lying electronic states. The leading electronic configurations of the relevant electronic states are shown in the middle panel of Fig. 2. At symmetric $\left(C_{s}\right)$ structures, one can generate 2 different triplet states $\left({ }^{3} \mathrm{~A}^{\prime}\right.$ and $\left.{ }^{3} \mathrm{~A}^{\prime \prime}\right)$ and two open-shell singlets with the same orbital occupations. At low-symmetry structures, all electronic configurations of the same multiplicity can mix, giving rise to highly multi-configurational wavefunctions. This multi-configurational character and multiple closely lying electronic states pose a challenge for theory.

It is easy to rationalize why there are two nearly degenerate triplet states. At the dissociation limit, the $\mathrm{O}\left({ }^{3} \mathrm{P}\right)$ term comprises 9 triplet states $\left(3 p\right.$-states for each $\left.S_{z}\right)$. From these, one $p$ orbital (call it $\left.p_{z}(\mathrm{O})\right)$ can form a bonding combination with the $\pi$-orbitals of ethylene, while others yield non-bonding combinations. In the triplet state with doubly occupied $p_{z}(\mathrm{O})$, an antibonding orbital is occupied leading to repulsive state, while in two other triplets, differing in occupation of non-bonding lone pairs, there is bonding interaction between $\mathrm{O}$ and $\mathrm{C}$ (as clearly illustrated by the natural orbitals in Fig. 4 of Ref. 7). The singlet $\mathrm{A}^{\prime}$ states can also contain closed-shell configurations. The weight of the closed-shell configuration in the lowest singlet is comparable with the weights of open-shell configurations. This closed-shell configuration leads to bonding interactions between the two radical centers. The weight of this configuration and, consequently, bonding interactions increase at smaller CCO angles, leading to an important product, ethylene and propylene oxides.

Fig. 3 shows relevant MOs in the acetylene-derived diradical, $\mathrm{C}_{2} \mathrm{H}_{2} \mathrm{O}$. The electronic structure of diradicals derived from triple-bond compounds is different from that of $\mathrm{C}_{2} \mathrm{H}_{4} \mathrm{O}$. In the lowest triplet state, there are two planar isomers, $Z$ and $E$. The symmetry plane separates the orbitals into the $\pi\left(\mathrm{a}^{\prime \prime}\right)$ and $\sigma\left(\mathrm{a}^{\prime}\right)$ manifolds. The $\pi$-system and the orbital occupations are remarkably similar to the $\pi$-system of the allyl radical (see Fig. S5 in SI), giving rise to the CC and $\mathrm{CO}$ bond orders of $\sim 1.5$ and and an increased barrier to rotation. The $\sigma$ system comprises lone pairs on oxygen and carbon. While these orbitals do not contribute towards bonding in the triplet state, they are important for the analysis of excited states and transition properties because they are involved in the $\mathrm{A}^{\prime \prime}$ transitions.

\section{Theoretical methods and computational details}

Electronic degeneracies and multi-configurational character of the low-lying states of the Cvetanović diradicals make electronic structure calculations rather involved. Figure 4 shows all electronic configurations that can be generated by distributing 4 electrons in 3 orbitals. There are five $\mathrm{A}^{\prime}$ determinants that can give rise to closed- and open-shell ${ }^{1} \mathrm{~A}^{\prime}$ configurations. Of these, determinants (4) and (5) give rise to ${ }^{3} \mathrm{~A}^{\prime}$ state. Determinants (6)-(9) give rise to open-shell ${ }^{1} \mathrm{~A}^{\prime \prime}$ and ${ }^{3} \mathrm{~A}^{\prime \prime}$ states. In contrast to many previous studies, which have employed multi-reference methods based on complete-active-space self-consistent field (CASSCF) augmented by various treatments of dynamic correlation, here we explore an alternative approach, equation-of-motion coupled-cluster methods with single and double excitations (EOM-CCSD). ${ }^{27-29}$ By combining deliberately chosen references with various types of EOM operators, EOM-CC can describe a 


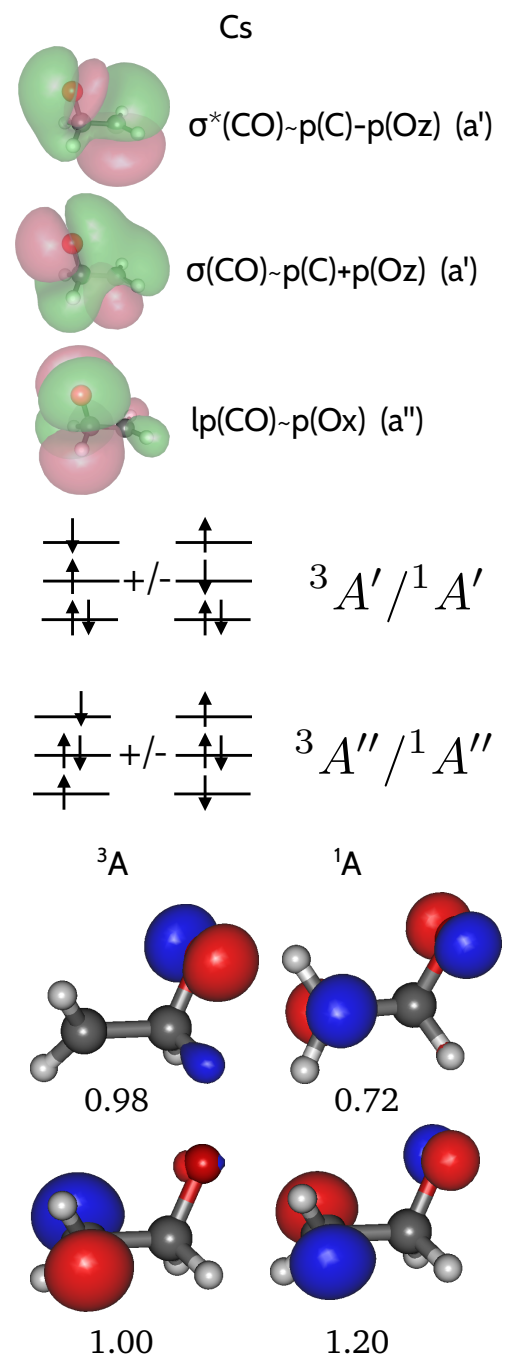

Figure 2: Top: RHF $/ 6-31 \mathrm{G}^{*}$ orbitals of the closed-shell ${ }^{1} \mathrm{~A}^{\prime}$ state computed at the ${ }^{3} \mathrm{~A}^{\prime \prime}$ optimized geometry $\left(\mathrm{C}_{s}\right)$. Middle: leading electronic configurations of the relevant electronic states. At low-symmetry geometries, all these configurations can mix, giving rise to heavily multiconfigurational wavefunctions. Bottom: Natural frontier orbitals and their occupations of the lowest singlet at triplet states at the optimized triplet geometry $\left(\mathrm{C}_{1}\right)$. 


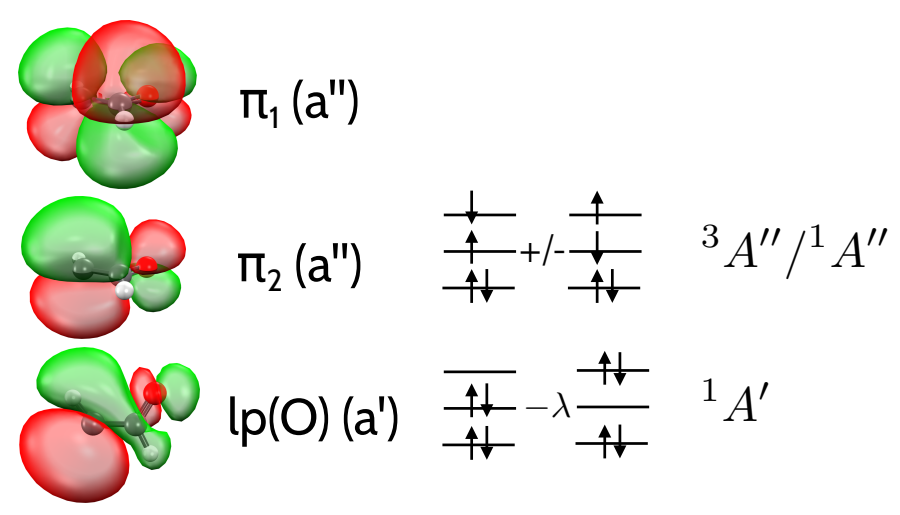

Figure 3: Left: $\mathrm{RHF} / 6-31 \mathrm{G}^{*}$ orbitals of the closed-shell state of the Z-isomer of the $\mathrm{C}_{2} \mathrm{H}_{2} \mathrm{O}$ diradical. Right: leading electronic configurations of the relevant electronic states.

wide range of multi-configurational wavefunctions within a single-reference formalism. Particularly attractive features of the EOM-CC methods are (i) simultaneous account of dynamic and non-dynamic correlation; (ii) their black-box nature (no active space selection or state averaging is involved); and (iii) their ability to describe multiple interacting states in the same calculation. The latter feature simplifies the calculation of interstate properties, such as spinorbit and non-adiabatic couplings, ${ }^{30,31}$ and the analysis of transition properties. ${ }^{32,33}$ However, despite its flexibility, some electronic degeneracy patterns are beyond the reach of currently available EOM-CC variants. ${ }^{34}$

The bottom panel of Fig. 4 shows electronic configurations, which are used as references in different EOM calculations. As one can see, only the DIP configurational space includes all 9 determinants on equal footing, thus providing the most balanced description of the electronic states. In contrast, in EE calculations, determinant (1) represents the reference and determinants (4)-(7) are single excitations and the rest are double excitations. The analysis of the singlet CCSD wavefunction reveals that closed-shell reference is ill-behaved near the lowest triplet minimum, i.e., the norms of the cluster amplitudes exceed unity. Consequently, EOMEE-CCSD leads to an unbalanced treatment of configurations and an incorrect state ordering. Moreover, the results of $(\mathrm{T})$ calculations are quite wrong; however, $(\mathrm{dT})$ and $\mathrm{f}(\mathrm{T})$ corrections do give consistent and reasonable results (see SI, Section 2). In EOM-SF, the set of target determinants depends on which triplet reference is used.

We assess the validity of different EOM models by comparing them with each other. As expected, EOM-SF and EOM-DIP yields similar results, while EOM-EE produces a different state ordering. EOM-SF treats the determinants of the target ${ }^{1} \mathrm{~A}^{\prime}$ states in a balanced manner (in this case the occupation of orbitals is approximately the same as in the reference triplet). However, in the target ${ }^{1} \mathrm{~A}^{\prime \prime}$ states the treatment of leading determinants is unbalanced: from ${ }^{3} \mathrm{~A}^{\prime \prime}$ reference determinant (4) is generated by a single excitation, whereas its spin-complete partner (5) cannot be obtained by a single excitation; the same is true for the ${ }^{1} \mathrm{~A}^{\prime}$ reference and determinants (6) and (7). The wavefunctions of these $\mathrm{A}^{\prime \prime}$ states are severely spin-contaminated, with $\left\langle S^{2}\right\rangle$ close to 1 . The PES (discussed in Section 4.1 and in SI) of this state lies in between singlet and triplet states from the EOM-DIP calculation, giving an averaged description.

To analyze the electronic wavefunctions, we employed libwfa library ${ }^{32}$ to compute natural orbitals (NOs) and natural transition orbitals (NTOs). NOs, defined as eigenvectors of a state 


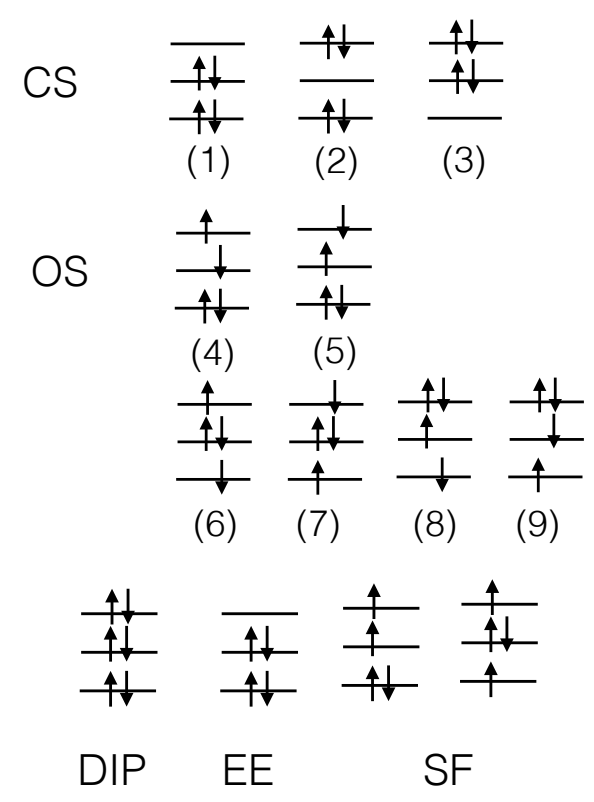

Figure 4: Top: Electronic configurations resulting from 4-electrons-in-3-orbitals pattern. Determinants (1)-(3) are of a closed-shell (CS) type and determinants (4)-(9) are of open-shell (OS) type. In ethylene $+\mathrm{O}$, at $\mathrm{C}_{s}$ structures, determinants (1)-(5) are of $\mathrm{A}^{\prime}$ symmetry, and determinants (6)-(9) are $\mathrm{A}^{\prime \prime}$. Bottom: electronic configurations of the reference determinants used in DIP, EE, and SF calculations.

one-particle density matrix,

$$
\rho_{p q} \equiv\left\langle\Psi\left|p^{\dagger} q\right| \Psi\right\rangle
$$

provide a compact representation of the wavefunction, facilitating the analysis of electronic states. ${ }^{35}$ NTOs are generalization of this concept to electronic transitions; they are defined as singular vectors of a one-particle transition density matrix $\gamma$ :

$$
\gamma_{p q}^{F I} \equiv\left\langle\Psi_{F}\left|p^{\dagger} q\right| \Psi_{I}\right\rangle
$$

where $\Psi_{I}$ and $\Psi_{F}$ denote the initial and final states. Corresponding left and right singular vectors form hole and particle pairs. Transition density matrix is diagonal in the NTO basis, thus, one-particle transition properties can be represented as matrix elements between the NTOs:

$$
\left\langle\Psi_{F}|A| \Psi_{I}\right\rangle=\operatorname{Tr}\left[A \gamma^{F I}\right]=\sum_{K} \sigma_{K}\left\langle\phi_{K}^{p}|A| \phi_{K}^{h}\right\rangle,
$$

where $\phi_{K}^{h}$ and $\phi_{K}^{p}$ are hole and particle pair corresponding to singular value $\sigma_{K}$. Very often, only a few singular values are non-vanishing and the sum can be approximated by a short truncated expansion. The sum of the squares of singular values gives the squared norm of the transition density matrix, $\|\gamma\|^{2}$.

We computed relevant stationary points on the lowest triplet state PES with CCSD/6-31G* and CCSD/cc-pVTZ. For acetylene- and propyne-derived species, the MECPs were computed ${ }^{36}$ with EOM-EE-CCSD /6-31G*. For ethylene and propene, the MECPs were computed by EOMDIP-CCSD /6-31G*. We used such a small basis set to avoid problems due to unstable dianionic reference. ${ }^{37}$ The calculations of MECPs are described in Section 3.1 below. All electronic structure calculations were carried out using the Q-Chem package. ${ }^{38,39}$ 


\subsection{Calculation and characterization of MECPs}

In NA-TST, MECPs play a role similar to those of transition states in adiabatic kinetics. ${ }^{23-25}$ We carried out MECP optimizations using the Lagrangian formalism defining the generalized gradient as the gradient on one of the states including the condition of the degeneracy. The description of the algorithm can be found in Ref. 36; additional details are given in SI. To validate the MECP, we analyzed the components of the gradients and the Hessians along the seam and perpendicular to it, using the criteria as in Ref. 40.

For well-behaved cases, MECP can be easily determined by optimization of the seam. We were able to locate MECP for acetylene- and propyne-derived diradicals by using this technique with and without constraint of the symmetry plane of the triplet geometries. To verify that the found stationary crossing points are minima, we performed frequency analysis along the seam (this procedure is analogous to the verification of minima versus saddle points in geometry optimization). We found that the symmetric structures have an out-of-plane imaginary mode of the effective Hessian (see Fig. S6 in SI). The frequencies at MECP for acetylene- and propynederived diradicals are given in SI. As expected from MECP properties (see Section 4 in SI), the gradients at the optimized MECP geometries are almost collinear. All angles are close to $180^{\circ}$, corresponding to the crossing of two parabolas, with separated minima and crossing in between these minima. The deviation of the angles between the gradients is larger for the propynederived diradical than for the acetylene-derived diradical, likely because of a larger number of degrees of freedom. For acetylene- and propyne-derived systems, the MECP geometries are close to the equilibrium geometry of the singlet diradical. Consequently, the MECP and the singlet minima are also close energetically (see Table S2 in the SI). Another observation is that the contribution of singlet Hessian dominates the effective Hessian and frequencies of MECP are singlet-like (Table S3 in SI).

In contrast, in the ethylene- and propene-derived diradicals, the MECPs are very close to the triplet state minima, both structurally and energetically. For these systems, optimization of MECP was non-trivial because of a very shallow PES and the lack of analytic gradients for EOM-DIP-CCSD (which for this system is the only EOM model capable of describing the relevant states around the MECP in a balanced way). We used the conjugate gradient method and steepest decent starting from the optimized triplet geometries. Often the optimization procedure converged to a stationary point, which has one or two imaginary frequency along the seam. In these cases, we located the true MECPs by applying displacements from these stationary points and rerunning the optimization. In these systems, the crossing coordinate at MECP is the bending of the OCC angle. 


\subsection{Evaluation of spin-orbit matrix elements}

The quantity relevant for the ISC rates calculations is the SOC constant (SOCC), ${ }^{41,42}$ which has the following form in spherical coordinates:

$$
\begin{aligned}
\left|\operatorname{SOCC}\left(s, s^{\prime}\right)\right|^{2} \equiv & \sum_{m_{s}=-s}^{s} \sum_{m_{s}^{\prime}=-s^{\prime}}^{s^{\prime}}\left[\left|\left\langle\Psi\left(s, m_{s}\right)\left|H_{-1}^{s o}\right| \Psi^{\prime}\left(s^{\prime}, m_{s}^{\prime}\right)\right\rangle\right|^{2}+\right. \\
& \left.\left|\left\langle\Psi\left(s, m_{s}\right)\left|H_{0}^{s o}\right| \Psi^{\prime}\left(s^{\prime}, m_{s}^{\prime}\right)\right\rangle\right|^{2}+\left|\left\langle\Psi\left(s, m_{s}\right)\left|H_{-1}^{s o}\right| \Psi^{\prime}\left(s^{\prime}, m_{s}^{\prime}\right)\right\rangle\right|^{2}\right]= \\
& \sum_{m_{s}=-s}^{s} \sum_{m_{s}^{\prime}=-s^{\prime}}^{s^{\prime}}\left|\left\langle\Psi\left(s, m_{s}\right)\left|H^{s o}\right| \Psi^{\prime}\left(s^{\prime}, m_{s}^{\prime}\right)\right\rangle\right|^{2}
\end{aligned}
$$

where $s, s^{\prime}$ are spins and $m_{s}, m_{s}^{\prime}$ are spin projections for the two states. We computed SOCCs using the EOM-CCSD wavefunctions. ${ }^{30}$ Calculations of SOCCs are complicated by several issues:

1. For non-spin-adapted wavefunctions (i.e., EOM-SF) spin contamination may affect the SOCC values.

2. Within the adiabatic approximation, the phases of electronic wavefunctions are not defined. Therefore, the phase changes randomly at different geometries or molecular orientations. This should be correctly taken care of when computing interstate matrix elements.

3. The phase consistency between the states of different spin projections in the same multiplet is important, because it defines the action of the ladder operators on the states and the transformation properties of the states. In the second quantization, the same applies to the components of tensor operators, such as the transition density matrix.

We tackled these issues as follows. The symmetry of one- and two-electron parts of the spin-orbit operator is the same as the symmetry of $\hat{\vec{L}} \cdot \hat{\vec{S}}$. Because dot product is rotationally invariant, we compute matrix elements in different molecular orientations and trace how the states change. Using properties of Wigner's D-matrices and the resolution-of-the-identity, one can establish the following relation:

$$
\begin{gathered}
\left\langle S M\left|H^{s o}\right| S^{\prime} M^{\prime}\right\rangle=\left\langle S M\left|R^{-1} R H^{s o} R^{-1} R\right| S^{\prime} M^{\prime}\right\rangle=\left\langle S M\left|R^{-1} H^{s o} R\right| S^{\prime} M^{\prime}\right\rangle= \\
\sum_{S, S_{z}^{\prime}}\left\langle S S_{z}\left|H^{s o}\right| S^{\prime} S_{z}^{\prime}\right\rangle\left(D_{S_{z}, M}^{(S)}\right)^{\dagger} D_{S_{z}^{\prime}, M^{\prime}}^{\left(S^{\prime}\right)}
\end{gathered}
$$

where $R$ is the rotation operator, $M$ denotes spin projections in the original coordinate system, and $S_{z}$ is a spin projection in the rotated coordinate system. In the case of singlet-triplet SOCC, $D_{0,0}^{0}=1$.

One can compute matrix elements $\left\langle 00\left|H^{S O}\right| 10\right\rangle$ by EOM-EE (as reference-EOM matrix elements) and EOM-SF (as EOM-EOM matrix elements) or $\left\langle 00\left|H^{S O}\right| 11\right\rangle$ by EOM-SF (reference$\mathrm{EOM}$ ) at three different molecular orientations and solve the system of linear equations. By randomizing the signs of the SOC elements, we established that phase inconsistency affects the final result of SOCC when the latter strategy is used. The same test yields identical values of SOCCs if $\left\langle 00\left|H^{S O}\right| 10\right\rangle$ is computed. 
We estimated the effect of spin contamination on the EOM-SF SOCCs for ethylene and propylene adducts by using a simple procedure described in Sec. 3 of SI. We found that the effect is small and the corrected SOCC values differ from the raw SOCC values by $1 \mathrm{~cm}^{-1} \mathrm{or}^{-}$ less (Table S1 in SI).

\subsection{Calculation of ISC rates}

In NA-TST, the probability of the ISC step is evaluated using the Landau-Zener (LZ) formula, which gives the probability of staying on the initial diabatic state (i.e., triplet PES):

$$
P=\exp \left(\frac{-2 \pi \mathrm{SOCC}^{2}}{v\left|s_{1}-s_{2}\right|}\right),
$$

where $v$ is a velocity and $s_{1}$ and $s_{2}$ are the slopes of the two potential energy curves. Tunneling can be included through a one-dimensional Wentzel-Kramers-Brillouin (WKB) model, which gives an approximate local overlap of the nuclear wavefunctions. Details can be found in Ref. 43.

We modeled the kinetics of ISC by applying NA-TST ${ }^{23}$ using the LZ formula, Eq. (6). We carried out these calculations using the open-source master equation code MESMER, which has been described in detail previously. ${ }^{24}$ For the sake of simplicity, we focused on the canonical formalism. Rovibrational densities of states were computed using the rigid-rotor harmonicoscillator approximation. MESMER input files with full details of the calculations are given in SI.

\section{Results and discussion}

\subsection{Relevant structures, potential energy scans, and states ordering}

The first question is the energy ordering of the relevant states and their structures. The triplet states can be well described by the CCSD method. Therefore, we used CCSD/cc-pVTZ for geometry optimizations. Fig. 5 shows optimized structures of the lowest triplet state of $\mathrm{C}_{2} \mathrm{H}_{4} \mathrm{O}$ and $\mathrm{C}_{3} \mathrm{H}_{5} \mathrm{O}$. The equilibrium structures are $C_{1}$. For $\mathrm{C}_{2} \mathrm{H}_{4} \mathrm{O}$, the structures optimized under $C_{s}$ constraint are very close to the $C_{1}$ one, both geometrically and energetically (these structures are shown in SI). In the propene-derived diradical, oxygen can attach to either terminal carbon or to the middle carbon atom, giving rise to nearly isoenergetic TC1 and TC2 isomers. For each structure, there are two conformers separated by relatively small barriers (about $1 \mathrm{kcal} / \mathrm{mol}$ ). Fig. 6 shows two lowest-energy structures of the $\mathrm{C}_{2} \mathrm{H}_{2} \mathrm{O}$ diradical $\left({ }^{3} \mathrm{~A}^{\prime \prime}\right)$. Both structures are true minima. The barrier for rotation is about $7 \mathrm{kcal} / \mathrm{mol}$.

It is unclear if there are stable minima on the singlet PES of the diradicals derived from ethylene and propylene. Our attempts to optimize the singlet structure by CCSD was unsuccessful. EOM-SF-CCSD and EOM-DIP-CCSD optimizations yield structures that might correspond to a very shallow minimum; tiny displacements lead to hydrogen shifts toward aldehyde or keton products.

Fig. 7 shows potential energy profiles of the lowest electronic states of $\mathrm{C}_{2} \mathrm{H}_{4} \mathrm{O}$ and $\mathrm{C}_{2} \mathrm{H}_{2} \mathrm{O}$ along torsional motion of the $\mathrm{CH}_{2}$ group. In $\mathrm{C}_{2} \mathrm{H}_{4} \mathrm{O}$ the triplet surface exhibits a rather small barrier (about $1 \mathrm{kcal} / \mathrm{mol}$ ) for internal rotation of the $\mathrm{CH}_{2}$ group. The rotation in the 


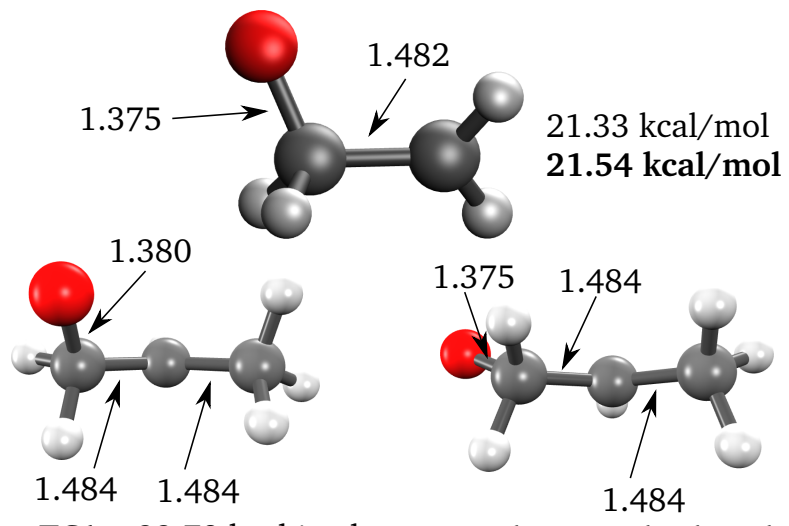

TC1a: $23.78 \mathrm{kcal} / \mathrm{mol} \quad$ TC1b: $23.27 \mathrm{kcal} / \mathrm{mol}$
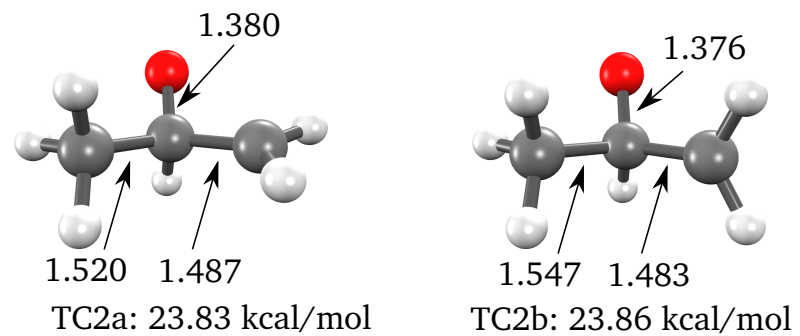

Figure 5: Equilibrium structures of the lowest triplet states of $\mathrm{C}_{2} \mathrm{H}_{4} \mathrm{O}$ (top) and $\mathrm{C}_{3} \mathrm{H}_{5} \mathrm{O}$ (bottom) computed with CCSD/cc-pVTZ. Energy relative to the reactants $\left(\mathrm{RH}+\mathrm{O}\left({ }^{3} \mathrm{P}\right)\right)$ is shown under each structure (plain text: CCSD/cc-pVTZ; bold: CCSD(dT)/cc-pVTZ; ZPE is not included). Bond lengths are in $\AA$. 


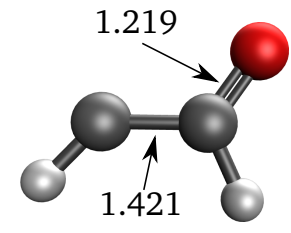

TE

$48.39 \mathrm{kcal} / \mathrm{mol}$

$49.07 \mathrm{kcal} / \mathrm{mol}$

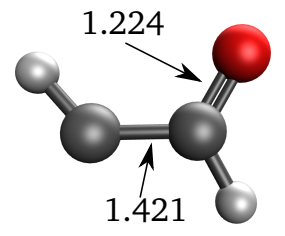

$\mathrm{TZ}$

$49.55 \mathrm{kcal} / \mathrm{mol}$

$50.57 \mathrm{kcal} / \mathrm{mol}$

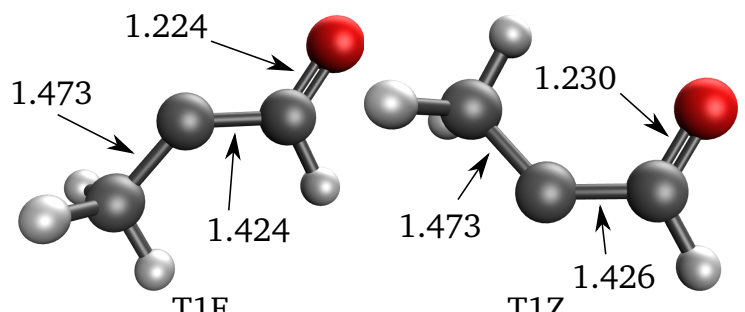

$\mathrm{T} 1 \mathrm{E}$

$49.47 \mathrm{kcal} / \mathrm{mol}$

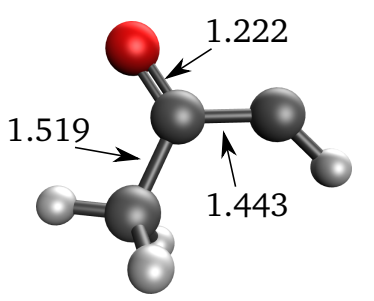

T2E

$51.36 \mathrm{kcal} / \mathrm{mol}$

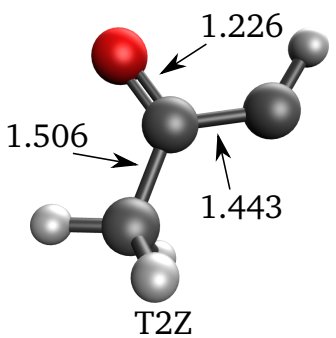

$48.25 \mathrm{kcal} / \mathrm{mol}$

Figure 6: $\mathrm{Z}$ and $\mathrm{E}$ isomers of the ${ }^{3} \mathrm{~A}^{\prime \prime}$ state of the $\mathrm{C}_{2} \mathrm{H}_{2} \mathrm{O}$ diradical. Energy relative to the reactants $\left(\mathrm{RH}+\mathrm{O}\left({ }^{3} \mathrm{P}\right)\right)$ is shown under each structure (plain text: CCSD/cc-pVTZ; bold: $\operatorname{CCSD}(\mathrm{dT}) /$ cc-pVTZ; ZPE is not included). Bond lengths are in $\AA$. 
triplet $\mathrm{C}_{2} \mathrm{H}_{4} \mathrm{O}$ is nearly free because there is no additional $\mathrm{CC}$ bonding ( $\mathrm{CC}$ bond length is close to the single bond in ethane). Due to the Pauli principle, the two unpaired electrons in the triplet state cannot occupy the same bonding MO, leading to non-bonding interactions between the 2 radical centers. The rotation leads to the crossing with the lowest singlet surface, giving an upper bound of the MECP energy of $0.05 \mathrm{eV}$. The singlet PESs show much larger variations along the torsional coordinate. Importantly, at large dihedral angles the two singlets are scrambled and one cannot correlate the individual states with the ${ }^{1} \mathrm{~A}^{\prime}$ or ${ }^{1} \mathrm{~A}^{\prime \prime}$ states. That is, almost all configurations from Fig. 4 are present in the respective wavefunctions. At the symmetric geometry, closed-shell configuration (1) from Fig. 4, which contributes to bonding between the radical centers, has a weight comparable to the open-shell determinants. As the ring at symmetric geometry closes, the energy drops and the weight of determinant (1) becomes dominant. We also note that the gap between the two triplets remains nearly constant $(\sim 0.2$ $\mathrm{eV}$. The vertical state orderings at the selected geometries is given in SI.

The triplet PES of the acetylene-derived diradical (Fig. 7, bottom) is very different: the barrier is an order of magnitude higher than in $\mathrm{C}_{2} \mathrm{H}_{4} \mathrm{O}$. This difference can be explained by the orbital pattern: the $\pi$-subsystem of the triplet state resembles that of the allyl radical, giving rise to an increased $\mathrm{CC}$ bond order (which is also manifested by a shorter $\mathrm{CC}$ distance). The gap between the triplet and the lowest singlet is also larger than in $\mathrm{C}_{2} \mathrm{H}_{4} \mathrm{O}$ (see $\mathrm{SI}$ for the state orderings at selected geometries). The character of the singlet state depends on geometry: near the triplet ${ }^{3} \mathrm{~A}^{\prime \prime}$ minimum this state has an open-shell ${ }^{1} \mathrm{~A}^{\prime \prime}$-like character, but in the structures with rotated $\mathrm{CH}$, it acquires a closed-shell character. The scans (Fig. 7) suggest a higher-lying singlet-triplet MECP, which is confirmed by the calculations.

\subsection{MECPs and SOCs: Cumulative analysis and implications for ISC rates}

Table 1 and 2 show relevant energy gaps and SOCCs. As explained in Section 3.1, the calculations of MECPs in these species were far from trivial. The triplet propylene adducts are nearly isoenergetic, differing by less than $1 \mathrm{kcal} / \mathrm{mol}$. At the optimized triplet geometries, the singlet is $2.3 \mathrm{kcal} / \mathrm{mol}$ above the triplet state. The MECP heights relative to the triplet minima are very small, i.e., less than $1 \mathrm{kcal} / \mathrm{mol}$.

All SOCCs for the propylene adduct (the arithmetic mean is $12.03 \mathrm{~cm}^{-1}$ and $15.00 \mathrm{~cm}^{-1}$ for the triplet geometries and the MECP respectively) are smaller than for the ethylene adduct, indicative of less efficient ISC, which qualitatively agrees with the experimentally observed smaller yield of singlet products in propylene.

For the adducts derived from acetylene and propyne, the difference in isomer energetics is slightly larger, up to $3 \mathrm{kcal} / \mathrm{mol}$. As discussed above, the electronic structure of these adducts is very different from ethylene-derived species and vertical singlet-triplet energy gaps are greater than $20 \mathrm{kcal} / \mathrm{mol}$. There is a significant difference between the MECP heights in acetylenederived and propyne-derived intermediates, sometimes up to a factor of two. This result is also consistent with the experimental finding: the branching ratio indicates much smaller yield of the singlet products in $\mathrm{C}_{2} \mathrm{H}_{2} \mathrm{O}$ than in $\mathrm{C}_{2} \mathrm{H}_{4} \mathrm{O}$. The difference between the branching ratios in the propyne $+\mathrm{O}$ and acetylene $+\mathrm{O}$ reactions can be explained by the MECP heights as well: smaller yields of singlet products in the acetylene+O reaction can be attributed to higher MECPs relative to propyne. 

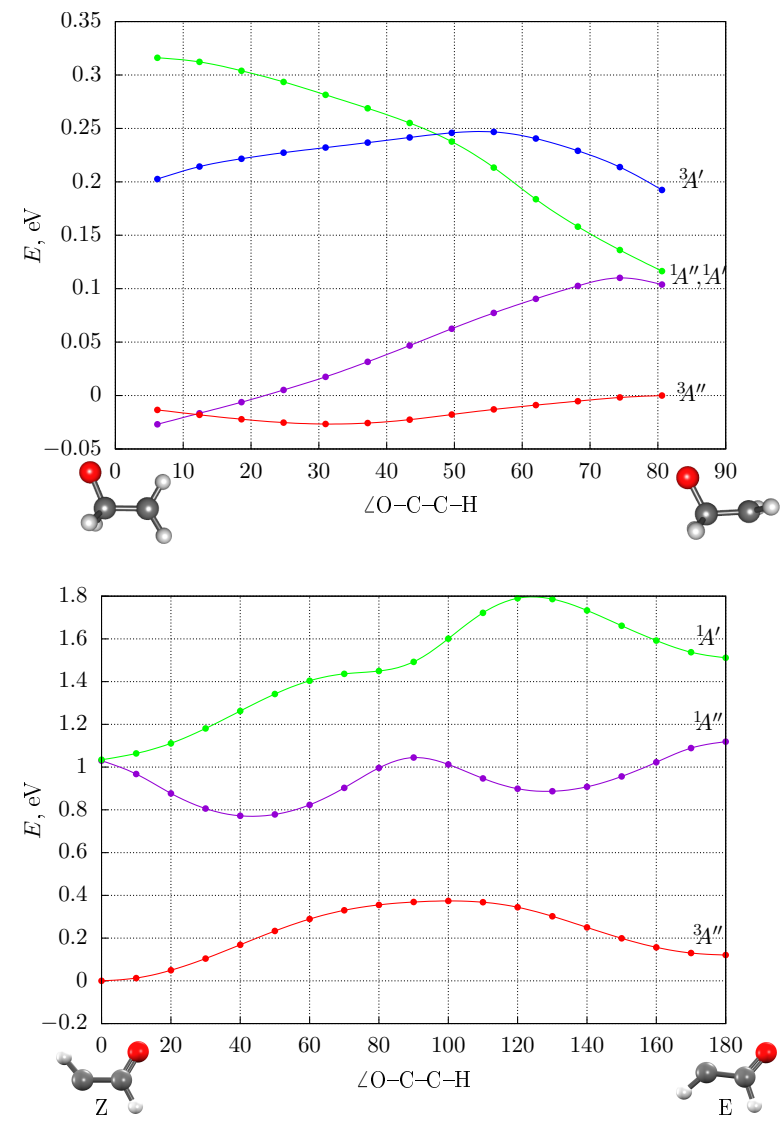

Figure 7: EOM-DIP-CCSD/6-31G* energies of low-lying states of $\mathrm{C}_{2} \mathrm{H}_{4} \mathrm{O}$ (top) and $\mathrm{C}_{2} \mathrm{H}_{2} \mathrm{O}$ (bottom) along torsional coordinate. The coordinates for the scans were obtained by constrained optimization of the lowest triplet state (CCSD/6-31G*). Symmetry labels correspond to the states computed at the symmetric structures (see Figs. 2 and 3). 
Table 1: Relevant energy differences $(\mathrm{kcal} / \mathrm{mol})$ for the lowest triplet states of prototypical Cvetanović diradicals.

\begin{tabular}{|l|llll|}
\hline Molecule & $\Delta E_{f}^{a}$ & $\Delta E^{b}$ & $\Delta E_{S T}^{c}$ & MECP $^{d}$ \\
\hline Ethylene & & & & \\
T & 20.43 & 0.0 & 1.36 & 0.06 \\
\hline Propene & & & & \\
TC1a & 23.40 & 0.08 & 1.84 & 0.24 \\
TC1b & 22.89 & 0.59 & 0.78 & 0.05 \\
TC2a & 22.81 & 0.0 & 1.90 & 0.35 \\
TC2b & 22.79 & 0.02 & 2.32 & 0.29 \\
\hline Acetylene & & & & \\
TZ & 47.85 & 0 & $23.74\left(\mathrm{~A}^{\prime \prime}\right) ; 23.82\left(\mathrm{~A}^{\prime}\right)$ & 10.39 \\
TE & 49.03 & 1.18 & $23.03\left(\mathrm{~A}^{\prime \prime}\right) ; 32.01\left(\mathrm{~A}^{\prime}\right)$ & 11.57 \\
\hline Propyne & & & & \\
T1E & 49.40 & 1.91 & $23.92\left(\mathrm{~A}^{\prime \prime}\right) ; 31.81\left(\mathrm{~A}^{\prime}\right)$ & 7.76 \\
T1Z & 51.31 & 0 & $25.01\left(\mathrm{~A}^{\prime \prime}\right) ; 28.88\left(\mathrm{~A}^{\prime}\right)$ & 9.67 \\
T2E & 48.20 & 3.11 & $28.20\left(\mathrm{~A}^{\prime \prime}\right) ; 32.12\left(\mathrm{~A}^{\prime}\right)$ & 7.52 \\
T2Z & 50.37 & 0.94 & $28.29\left(\mathrm{~A}^{\prime \prime}\right) ; 27.33\left(\mathrm{~A}^{\prime}\right)$ & 9.68 \\
\hline
\end{tabular}

${ }^{a}$ Energy relative to the $\mathrm{RH}+\mathrm{O}\left({ }^{3} \mathrm{P}\right)$ asymptote, $\mathrm{CCSD} / \mathrm{cc}-\mathrm{pVTZ}$.

${ }^{b}$ Energy relative to the lowest isomer for each species.

${ }^{c}$ Vertical singlet triplet energy gap $\left(E_{T}-E_{S}\right)$, EOM-DIP-CCSD /6-31G*.

${ }^{d}$ MECP location relative to the equilibrium structure of each isomer.

Table 2: SOCCs $\left(\mathrm{cm}^{-1}\right)$ at equilibrium triplet geometries and at MECPs.

\begin{tabular}{|l|l|l|}
\hline Molecule & SOCC at T & SOCC at MECP \\
\hline $\begin{array}{l}\text { Ethylene } \\
\text { T }\end{array}$ & 17.14 & 14.28 \\
\hline $\begin{array}{l}\text { Propene } \\
\text { TC1a }\end{array}$ & 13.68 & 17.37 \\
TC1b & 13.33 & 10.54 \\
TC2a & 16.56 & 18.27 \\
TC2b & 4.55 & 13.81 \\
\hline Acetylene & & \\
TZ & $0.27\left(A^{\prime \prime}\right) ; 10.52\left(A^{\prime}\right)$ & 13.53 \\
TE & $0.32\left(A^{\prime \prime}\right) ; 7.13\left(A^{\prime}\right)$ & 13.53 \\
\hline Propyne & & \\
T1E & $0.24\left(A^{\prime \prime}\right) ; 1.43\left(A^{\prime}\right)$ & 15.07 \\
T1Z & $0.17\left(A^{\prime \prime}\right) ; 8.17\left(A^{\prime}\right)$ & 15.07 \\
T2E & $0.26\left(A^{\prime \prime}\right) ; 7.81\left(A^{\prime}\right)$ & 16.67 \\
T2Z & $0.36\left(A^{\prime \prime}\right) ; 7.17\left(A^{\prime}\right)$ & 16.67 \\
\hline
\end{tabular}

${ }^{a}$ Coupling with the lowest singlet. 


\subsection{Analysis of SOCs and extended El-Sayed's rules}

El-Sayed's rules ${ }^{44}$ predict different magnitude of SOCs for the two triplet states in $\mathrm{C}_{2} \mathrm{H}_{4} \mathrm{O}$ : the coupling between ${ }^{3} \mathrm{~A}^{\prime \prime}$ and ${ }^{1} \mathrm{~A}^{\prime}$ is expected to be much larger than that between ${ }^{3} \mathrm{~A}^{\prime}$ and ${ }^{1} \mathrm{~A}^{\prime}$ or between ${ }^{3} \mathrm{~A}^{\prime \prime}$ and ${ }^{1} \mathrm{~A}^{\prime \prime}$ because rotation of the $p(\mathrm{O})$ orbital is needed for significant coupling. The calculations yield two orders of magnitude difference between $\mathrm{SOCC}\left({ }^{3} \mathrm{~A}^{\prime \prime},{ }^{1} \mathrm{~A}^{\prime}\right)$ and $\operatorname{SOCC}\left({ }^{3} \mathrm{~A}^{\prime \prime},{ }^{1} \mathrm{~A}^{\prime \prime}\right)$, whereas $\operatorname{SOCC}\left({ }^{3} A^{\prime},{ }^{1} A^{\prime}\right)$ has an intermediate value. To rationalize these couplings, we use El-Sayed's rule and NTOs. Figs. 8 and 9 show the NTOs, norms of oneparticle transition density matrix, and SOCCs for several states in ethylene-derived diradical and for the lowest triplet and singlet states in various isomers and conformers of the propylenederived diradicals.

Fig. 8 shows that for the ${ }^{3} A^{\prime \prime},{ }^{1} A^{\prime \prime}$ transition, there is no rotation of hole and particle orbitals, giving rise to a weak coupling. Note a similarity between the holes and particles: there is no change in orbital shape, and these two states can be represented well by spin-adapted configurations of two open-shell determinants constructed from the respective NTOs. There is always a sign change in these combinations, coming from the Clebsh-Gordon coefficients, which can be observed in one of the NTO pairs for $\left({ }^{3} \mathrm{~A}^{\prime \prime},{ }^{1} \mathrm{~A}^{\prime \prime}\right)$, because singular value decomposition preserves phases. ${ }^{1} \mathrm{~A}^{\prime},{ }^{3} \mathrm{~A}^{\prime \prime}$ transition leads to $90^{\circ}$ rotation of $p(\mathrm{O})$, giving rise to large coupling. The ${ }^{3} \mathrm{~A}^{\prime},{ }^{1} \mathrm{~A}^{\prime}$ transition leads to partial rotation of $p(\mathrm{O})$ and $p(\mathrm{C})$ in the plane of symmetry. The angle of this rotation is about $30^{\circ}$. In SI, we extend this analysis and illustrate that an intermediate value of SOCC corresponds to partial rotation of NTOs.

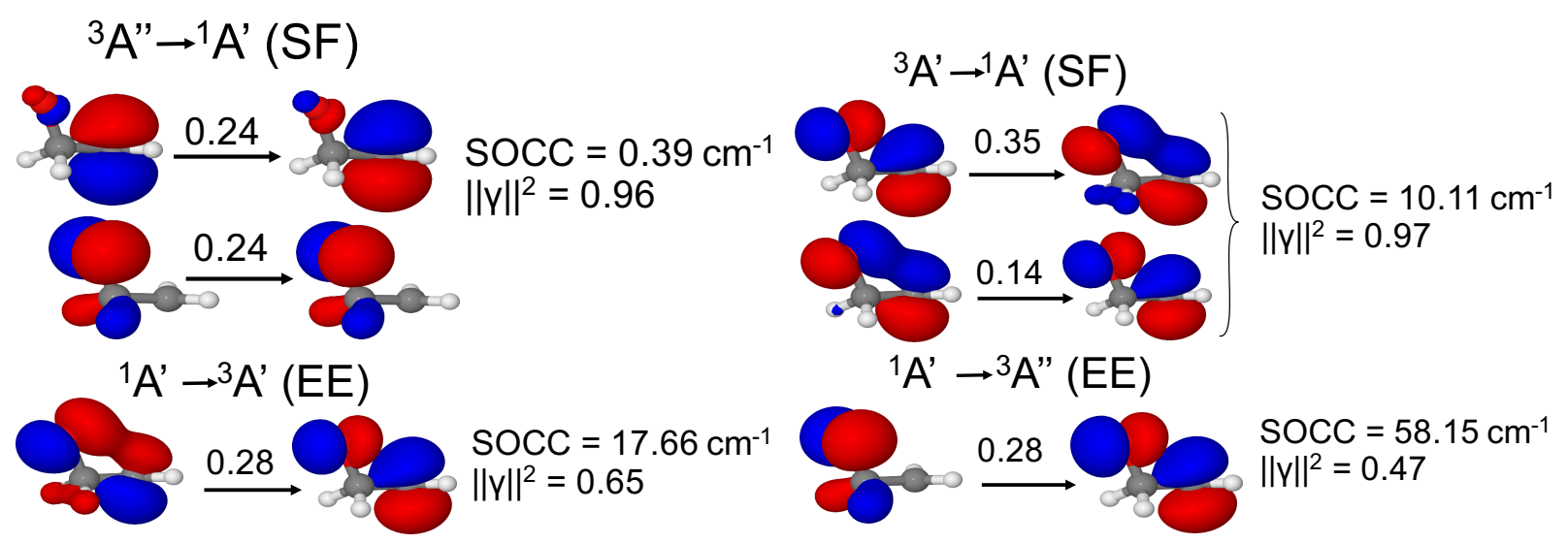

Figure 8: NTOs and SOCCs between the singlet and triplet states at symmetric $\mathrm{C}_{2} \mathrm{H}_{4} \mathrm{O}$ geometry computed using EOM-SF-CCSD/6-31G* and EOM-EE-CCSD/6-31G* wavefunctions. Only alpha orbitals are shown. NTO analysis was performed using $A \rightarrow B$ transition matrix.

\subsection{Implications for ISC rates}

Although full rate and branching ratios require complicated multi-well calculations, some qualitative predictions can be made by simply considering the LZ formula, Eq. (6). This expression indicates that the non-adiabatic (here, triplet-singlet) transitions are suppressed when the velocity of crossing is high. Since the MECPs heights in the ethylene- and propylene-derived diradicals are small, the ISC is likely to proceed as follows: oxygen attaches to one of the carbons, the intermediate collides with other molecules and loses ro-vibrational energy, low 

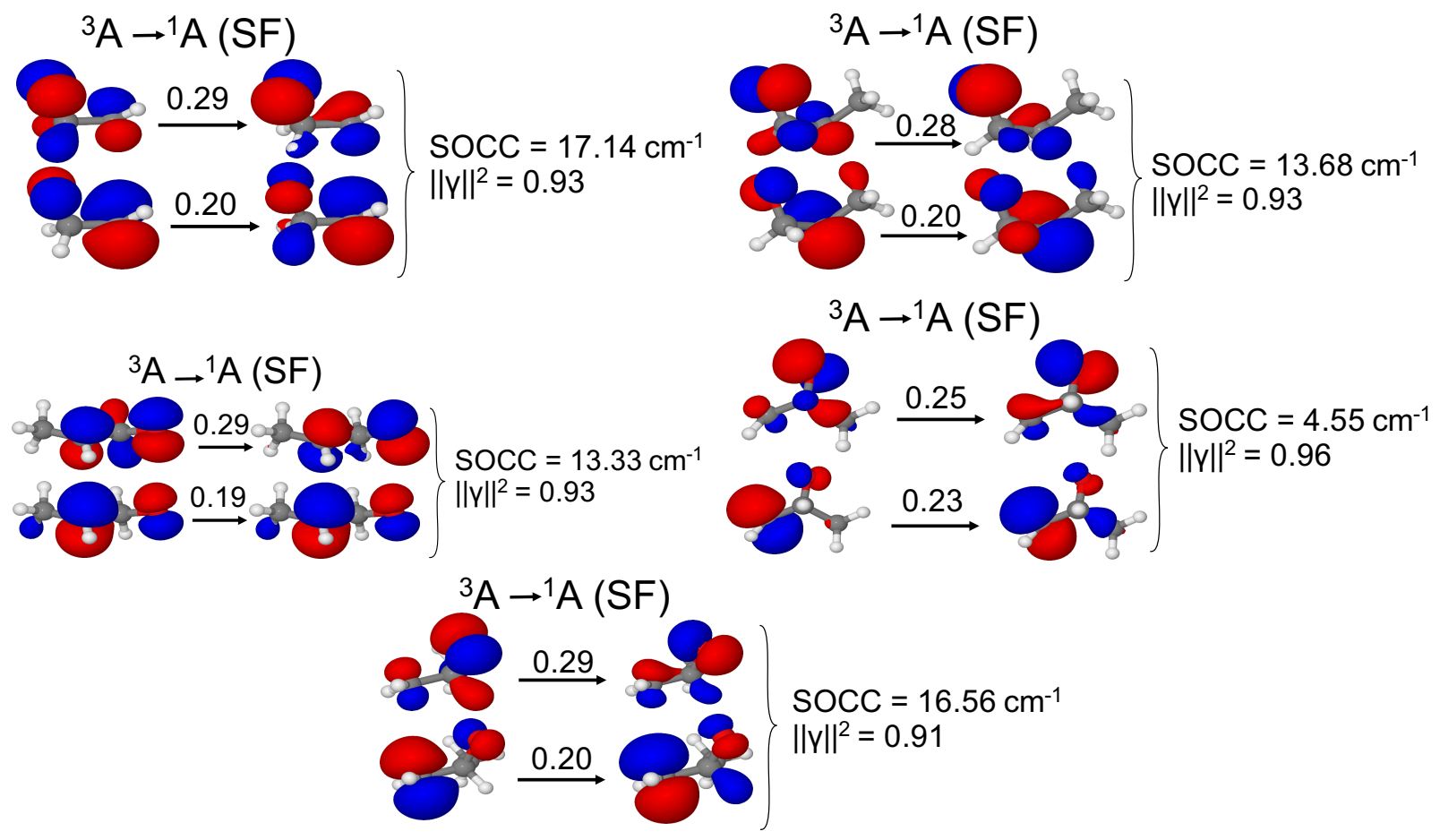

Figure 9: NTOs and SOCCs between the lowest triplet and singlet states at symmetric triplet minimum geometries of ethylene-derived intermediate and TC1a, TC1b, TC2a, TC2b propylene-derived intermediate, computed by EOM-SF-CCSD/6-31G* . Only alpha orbitals are shown. NTO analysis was performed using $A \rightarrow B$ transition matrix. 
Table 3: Canonical ISC rates $^{a}\left(\mathrm{~s}^{-1}\right)$ at $300 \mathrm{~K}$ (high-pressure limit).

\begin{tabular}{|l|ll|}
\hline Molecule & LZ rate & WKB rate \\
\hline Ethylene & & \\
T & $1.2 \cdot 10^{10}$ & $5.67 \cdot 10^{9}$ \\
\hline Propene & & \\
TC1a & $3.4 \cdot 10^{10}$ & $2.7 \cdot 10^{10}$ \\
TC1b & $2.0 \cdot 10^{10}$ & $1.2 \cdot 10^{10}$ \\
TC2a & $1.0 \cdot 10^{10}$ & $9.9 \cdot 10^{9}$ \\
TC2b & $8.4 \cdot 10^{9}$ & $8.2 \cdot 10^{9}$ \\
\hline Acetylene & $1.9 \cdot 10^{4}$ & $2.0 \cdot 10^{4}$ \\
TZ & $2.1 \cdot 10^{3}$ & $3.5 \cdot 10^{3}$ \\
TE & $1.7 \cdot 10^{4}$ & $1.7 \cdot 10^{4}$ \\
\hline Propyne & $10.0 \cdot 10^{4}$ & $2.5 \cdot 10^{5}$ \\
T1E & $4.1 \cdot 10^{4}$ & $6.3 \cdot 10^{4}$ \\
T1Z & $9.7 \cdot 10^{2}$ & $1.5 \cdot 10^{3}$ \\
T2E & $5.7 \cdot 10^{4}$ & $1.8 \cdot 10^{5}$ \\
T2Z & $1.4 \cdot 10^{3}$ & $4.4 \cdot 10^{3}$ \\
\hline
\end{tabular}

a All-harmonic mode approximation was used for all the rates reported above. The total rate of ISC (shown in the same row as acetylene, propyne, etc) is a sum of the rates of from all intermediates.

vibrational energy encourages ISC (by virtue of the LZ rule) through a small barrier from CCO bending mode (crossing coordinate from MECP calculations, see Fig. S7 in SI) or through a small barrier by internal rotation. This mechanism is different from the one originally proposed by Cvetanović, who assumed ISC of the hot intermediate, followed by collisional stabilization of the singlet intermediate. Considering the overall shape of the PES, the triplet intermediate acts as a trap: once the system is there, the relaxation due to collisions closes pathways on the triplet surface due to high barriers, while the ISC channel remains competitive owing to low MECP heights.

As described in Section 3.3 we modeled the kinetics of ISC in these systems by applying NA$\mathrm{TST}^{23}$ in its microcanonical form. By using MESMER software, we calculated high-pressure limiting ISC rate coefficients for each triplet species; these are given in Table 3. These rate coefficients agree qualitatively with the experimental branching ratios ${ }^{16-19}$ shown in Figure 1 and illustrate the relative ISC rates between the different systems. For example, the computed rates are very close for the ehtene and propene-derived species, whereas in the triple-bond adducts the methyl group leads to a 5-10-fold rate increase, consistently with a significant increase in the yield of singlet products in propyne relative to acenylene. The modelled highpressure conditions correspond to ultrafast vibrational relaxation, often observed in condensed phase. Interestingly, in the experiments studying oxygen addition to double-bond species ${ }^{2}$ in liquid nitrogen or in solid films the fragmentation products have not been observed. This is consistent with our mechanism: once a triplet intermediate is formed, it is cooled down, making dissociative pathways energetically inaccessible while activating ISC. We note that under combustion conditions this thermal, high-pressure-limit picture can be misleading. In reality, the Cvetanović diradicals are formed with excess internal energy and the ISC (and other 
reactive channels on the triple surface) compete with collisional energy transfer with the bath.

\section{Conclusion}

We characterized four prototypical Cvetanović diradicals, $\mathrm{C}_{2} \mathrm{H}_{4} \mathrm{O}, \mathrm{C}_{3} \mathrm{H}_{6} \mathrm{O}, \mathrm{C}_{2} \mathrm{H}_{2} \mathrm{O}$, and $\mathrm{C}_{3} \mathrm{H}_{4} \mathrm{O}$, with an emphasis on the electronic structure aspects relevant to the ISC rates. We found that methylation has a relatively small effect on the electronic structure. In contrast, the diradicals derived from double- and triple-bond unsaturated hydrocarbons are very different.

In ethylene-derived diradicals, there are four closely lying electronic states differing by the occupations of oxygen's $p$-orbitals. The lowest singlet state is multi-configurational and the weights of different configurations depend on the geometry. While its diradical structure was described long time ago, ${ }^{2}$ the exact shape and orientation of orbitals can be established only by accurate correlated calculations. The NTO analysis using SF wavefunctions confirms openshell nature of the singlet states and serves as a basis for the extension of El-Sayed's rules. The lowest triplet state features a very shallow PES profile along the torsional coordinate, with a low-energy $(\sim 1 \mathrm{kcal} / \mathrm{mol})$ MECP located close to the equilibrium triplet structure. There is no stable minima corresponding to the singlet diradical.

In contrast to ethylene, in the acetylene-derived adducts the barriers for the internal rotation and singlet-triplet MECPs are much higher. Moreover, these species have stable closed-shell singlet diradical (its structure is close to MECP). Because the singlet geometry is MECP-like, the difference in MECP heights in the acetylene- and propyne-derived species correlates with the adiabatic singlet-triplet gaps.

The magnitude of SOCCs depends strongly on the molecular structure, which can be explained by El-Sayed's rules. In the acetylene/propyne adducts, the magnitude of SOCCs at MECP is almost 10 times larger than at the equilibrium structures.

The calculated MECPs heights and SOCCs reveal that the different effect of methyl group on the ISC rates in the double-bond compounds versus the triple-bond ones can be attributed to different mechanisms. In the former, the MECP is very low and the rate is controlled by the SOCC variations, whereas in the latter the MECP is high and the main effect due to methyl substitutions is explained by the variations in MECP heights. Our study also highlights the great complexity of these small species, drawing attention to the methodological challenges and possible pitfalls in theoretical modeling of their reactivity.

\section{Acknowledgment}

We are grateful to Dr. David Osborn (Sandia National Laboratory) for many stimulating and illuminating discussions. This work is supported by the U.S. Air Force of Scientific Research (AFOSR) under grant number FA9550-16-1-0051.

Supporting information is available: Computational details, relevant Cartesian geometries, MESMER input files, additional data on state ordering, SOCCs analysis. 


\section{References}

[1] Cvetanović, R. J. Evaluated chemical kinetic data for the reactions of atomic oxygen $\mathrm{O}\left({ }^{3} \mathrm{P}\right)$ with unsaturated hydrocarbons J. Phys. Chem. Ref. Data 1987, 16, 261-326.

[2] Cvetanović, R. J.; Singleton, D. L. Reaction of oxygen atoms with olefins Rev. Chem. Int. 1984, 5, 183-226.

[3] Taatjes, C. A.; Osborn, D. L.; Selby, T. M.; Meloni, G.; Trevitt, A. J.; Epifanovsky, E.; Krylov, A. I.; Sirjean, B.; Dames, E.; Wang, H. Products of the benzene $+\mathrm{O}\left({ }^{3} \mathrm{P}\right)$ reaction J. Phys. Chem. A 2010, 114, 3355-3370.

[4] Sabbah, H.; Biennier, L.; Sims, I.A.; Georgievskii, Y.; Klippenstein, S.J.; Smith, I.W.M. Understanding reactivity at very low temperatures: The reactions of oxygen atoms with alkenes Science 2007, 317, 102-105.

[5] Bergantini, A.; Abplanalp, M. J.; Pokhilko, P.; Krylov, A. I.; Shingledecker, C. N.; Herbst, E.; Kaiser, R. I. A combined experimental and theoretical study on the formation of interstellar propylene oxide $\left(\mathrm{CH}_{3} \mathrm{CHCH}_{2} \mathrm{O}\right)$ - a chiral molecule Astrophys. J. 2018; in press.

[6] Minaev, B.F.; Kukueva, V.V.; Bykov, A.S. The role of spin-orbit coupling in olefine combustion reaction of the triplet atomic oxygen with olefins Colloid. Czech. Chem. Commun 2004.

[7] Dupuis, M.; Wendoloski, J.J.; Takada, T.; Lester, W.A. Theoretical-study of electrophilic addition $-\mathrm{O}\left({ }^{3} \mathrm{P}\right)+\mathrm{C}_{2} \mathrm{H}_{4}$ J. Chem. Phys. 1982, 76, 481-487.

[8] Zhao, S.; Wu, W.; Zhao, H.; Wang, H.; Yang, C.; Liu, K.; Su, H. Adiabatic and nonadiabatic reaction pathways of the $\mathrm{O}\left({ }^{3} \mathrm{P}\right)$ with propyne J. Phys. Chem. A 2009, 113, 23-34.

[9] Fu, B.; Han, Y.-C.; Bowman, J.M.; Leonori, F.; Balucani, N.; Angelucci, L.; Occhiogrosso, A.; Petrucci, R.; Casavecchia, P. Experimental and theoretical studies of the $\mathrm{O}\left({ }^{3} \mathrm{P}\right)+$ $\mathrm{C}_{2} \mathrm{H}_{4}$ reaction dynamics: Collision energy dependence of branching ratios and extent of intersystem crossing J. Chem. Phys. 2012, 137, 22A532.

[10] Fu, B.; Han, Y.-C.; Bowman, J.M.; Angelucci, L.; Balucani, N.; Leonori, F.; Casavecchia, $\mathrm{P}$. Intersystem crossing and dynamics in $\mathrm{O}\left({ }^{3} \mathrm{P}\right)+\mathrm{C}_{2} \mathrm{H}_{4}$ multichannel reaction: Experiment validates theory Proc. Nat. Acad. Sci. 2012, 109, 9733-9738.

[11] Nguyen, T.L.; Vereecken, L.; Hou, X.J.; Nguyen, M.T.; Peeters, J. Potential energy surfaces, product distributions and thermal rate coefficients of the reaction of $\mathrm{O}\left({ }^{3} \mathrm{P}\right)$ with $\mathrm{C}_{2} \mathrm{H}_{4}\left(\mathrm{X}^{1} \mathrm{~A}_{g}\right)$ : A comprehensive theoretical study J. Phys. Chem. A 2005, 109.

[12] Hodgson, D.; Zhang, H.-Y.; Nimlos, M.R.; McKinnon, J.T. Quantum chemical and RRKM invesigation of the elementary channels of the reaction $\mathrm{C}_{6} \mathrm{H}_{6}+\mathrm{O}\left({ }^{3} \mathrm{P}\right)$ J. Phys. Chem. A 2001, 105, 4316-4327.

[13] Nguen, T.L.; Peeters, J.; Vereecken, L. Theoretical reinvestigation of the $\mathrm{O}\left({ }^{3} \mathrm{P}\right)+\mathrm{C}_{6} \mathrm{H}_{6}$ reaction: Quantum chemical and statistical rate calculations J. Phys. Chem. A 2007, 111, $3836-3849$. 
[14] West, A.C.; Lynch, J.D.; Sellner, B.; Lischka, H.; Hase, W.L.; Windus, T.L. O + $\mathrm{C}_{2} \mathrm{H}_{4}$ potential energy surface: Excited states and biradicals at the multireference level Theor. Chim. Acta 2012, 131, 1123.

[15] X.Li; Jasper, A.W.; Zádor, J.; Miller, J.A.; Klippenstein, S.J. Theoretical kinetics of O + $\mathrm{C}_{2} \mathrm{H}_{4}$ Proceedings of the Combustion Institute 2016, 36, 219-227.

[16] Casavecchia, P.; Capozza, G.; Segoloni, E.; Leonori, F.; Balucani, N.; Volpi, G. G. Dynamics of the $\mathrm{O}\left({ }^{3} \mathrm{P}\right)+\mathrm{C}_{2} \mathrm{H}_{4}$ reaction: Identification of five primary product channels (vinoxy, acetyl, methyl, methylene, and ketene) and branching ratios by the crossed molecular beam technique with soft electron ionization J. Phys. Chem. A 2005, 109, 3527-3530.

[17] Capozza, G.; Segoloni, E.; Leonori, F.; Volpi, G. G.; Casavecchia, P. Soft electron impact ionization in crossed molecular beam reactive scattering: The dynamics of the $\mathrm{O}\left({ }^{3} \mathrm{P}\right)+\mathrm{C}_{2} \mathrm{H}_{2}$ reaction J. Chem. Phys. 2004, 120, 4557-4560.

[18] Savee, J.D.; Welz, O.; Taatjes, C.A.; Osborn, D.L. New mechanistic insights to the O $\left({ }^{3} \mathrm{P}\right)$ + propene reaction from multiplexed photoionization mass spectrometry J. Chem. Phys. 2012, 14, 10410-10423.

[19] Savee, J. D.; Borkar, S.; Welz, O.; Sztar'a, B.; Taatjes, C. A.; Osborn, D. L. Multiplexed photoionization mass spectrometry investigation of the $\mathrm{O}\left({ }^{3} \mathrm{P}\right)+$ propyne reaction $J$. Phys. Chem. A 2015, 119, 7388-7403.

[20] Osborn, D. L. Reaction mechanisms on multiwell potential energy surfaces in combustion (and atmospheric) chemistry Annu. Rev. Phys. Chem. 2017, 68, 233-260.

[21] Vereecken, L.; Glowacki, D. R.; Pilling, M. J. Theoretical chemical kinetics in tropospheric chemistry: Methodologies and applications Chem. Rev. 2015, 115, 4063-4114.

[22] Marian, C.M. Spin-orbit coupling and intersystem crossing in molecules WIREs Comput. Mol. Sci. 2012, 2, 187-203.

[23] Harvey, J. N. Understanding the kinetics of spin-forbidden chemical reactions Phys. Chem. Chem. Phys. 2006, 200\%, 331-343.

[24] Glowacki, D. R.; Liang, C.-H.; Morley, C.; Pilling, M. J.; Robertson, S. H. MESMER: An open-source master equation solver for multi-energy well reactions J. Phys. Chem. A 2012, 116, 9545-9560.

[25] Lykhin, A. O.; Kaliakin, D. S.; dePolo, G. E.; Kuzubov, A. A.; Varganov, S. A. Nonadiabatic transition state theory: Application to intersystem crossings in the active sites of metal-sulfur proteins Int. J. Quant. Chem. 2016, 116, 750-761.

[26] West, A.C.; Kretchmer, J.S.; Sellner, B.; Park, K.; Hase, W.L.; Lischka, H.; Windus, T.L. $\mathrm{O}+\mathrm{C}_{2} \mathrm{H}_{4}$ potential energy surface: Study at the multireference level J. Phys. Chem. A 2009, 113, 12663-12674.

[27] Krylov, A. I. Equation-of-motion coupled-cluster methods for open-shell and electronically excited species: The hitchhiker's guide to Fock space Annu. Rev. Phys. Chem. 2008, 59, 433-462. 
[28] Sneskov, K.; Christiansen, O. Excited state coupled cluster methods WIREs Comput. Mol. Sci. 2012, 2, 566-584.

[29] Bartlett, R.J. Coupled-cluster theory and its equation-of-motion extensions WIREs Comput. Mol. Sci. 2012, 2, 126-138.

[30] Epifanovsky, E.; Klein, K.; Stopkowicz, S.; Gauss, J.; Krylov, A. I. Spin-orbit couplings within the equation-of-motion coupled-cluster framework: Theory, implementation, and benchmark calculations J. Chem. Phys. 2015, 143, 064102.

[31] Faraji, S.; Matsika, S.; Krylov, A. I. Calculations of non-adiabatic couplings within equation-of-motion coupled-cluster framework: Theory, implementation, and validation against multi-reference methods J. Chem. Phys. 2018, 148, 044103.

[32] Plasser, F.; Wormit, M.; Dreuw, A. New tools for the systematic analysis and visualization of electronic excitations. I. Formalism J. Chem. Phys. 2014, 141, 024106-13.

[33] Mewes, S.; Plasser, F.; Krylov, A. I.; Dreuw, A. Benchmarking excited-state calculations using exciton properties J. Chem. Theory Comput. 2018, 14, 710-725.

[34] Orms, N.; Krylov, A.I. Modeling photoelectron spectra of $\mathrm{CuO}, \mathrm{Cu}_{2} \mathrm{O}$, and $\mathrm{CuO}_{2}$ anions with equation-of-motion coupled-cluster methods: An adventure in Fock space J. Phys. Chem. A 2018, 122, 3653-3664.

[35] Orms, N.; Rehn, D. R.; Dreuw, A.; Krylov, A. I. Characterizing bonding patterns in diradicals and triradicals by density-based wave function analysis: A uniform approach $J$. Chem. Theory Comput. 2017, 14, 638-648.

[36] Epifanovsky, E.; Krylov, A. I. Direct location of the minimum point on intersection seams of potential energy surfaces with equation-of-motion coupled-cluster methods Mol. Phys. 2007, 105, 2515-2525.

[37] Kuś, T.; Krylov, A. I. Using the charge stabilization technique in the double ionization potential equation-of-motion calculations with dianion references J. Chem. Phys. 2011, 135, 084109.

[38] Shao, Y.; Gan, Z.; Epifanovsky, E.; Gilbert, A.T.B.; Wormit, M.; Kussmann, J.; Lange, A.W.; Behn, A.; Deng, J.; Feng, X., et al. Advances in molecular quantum chemistry contained in the Q-Chem 4 program package Mol. Phys. 2015, 113, 184-215.

[39] Krylov, A. I.; Gill, P. M. W. Q-Chem: An engine for innovation WIREs Comput. Mol. Sci. 2013, 3, 317-326.

[40] Harvey, J. N.; Aschi, M. Spin-forbidden dehydrogenation of methoxy cation: A statistical view Phys. Chem. Chem. Phys. 1999, 1, 5555-5563.

[41] Fedorov, D.G.; Gordon, M.S. A study of the relative importance of one and two-electron contributions to spin-orbit coupling J. Chem. Phys. 2000, 112, 5611-5623.

[42] Fedorov, D.G.; Koseki, S.; Schmidt, M.W.; Gordon, M.S. Spin-orbit coupling in molecules: Chemistry beyond the adiabatic approximation Int. Rev. Phys. Chem. 2003, 22, 551-592. 
[43] Harvey, J. N; Aschi, M. Modelling spin-forbidden reactions: recombination of carbon monoxide with iron tetracarbonyl Faraday Discuss. 2003, 124, 129-143.

[44] El-Sayed, M.A. Triplet state: Its radiative and non-radiative properties Acc. Chem. Res. 1968, 1, 8-16. 